\title{
Information support for the procedure of measuring the flow rate of water in open channels by a water current meter with Arduino Micro board
}

https://doi.org/10.31713/MCIT.2020.22

\author{
Serhii Klimov \\ dept. of Hydroinformatics \\ National University of Water and Environmental Engineering \\ Rivne, Ukraine \\ s.v.klimov@nuwm.edu.ua
}

\author{
Dmytro Reut \\ dept. of Computer Science and Applied Mathematics \\ National University of Water and Environmental Engineering \\ Rivne, Ukraine \\ d.t.reut@nuwm.edu.ua
}

\author{
Anastasiya Klimova \\ dept. of Licensing and Accreditation Department \\ National University of Water and Environmental Engineering \\ Rivne, Ukraine \\ a.v.klimova@nuwm.edu.ua
}

\begin{abstract}
The modernization of a typical water current meter is proposed to facilitate hydrological observations on natural and artificial open watercourses. An Arduino Pro Micro microcontroller board was used to read the speed of the current meter and primary data processing, programs were developed: for the lower level - a microcontroller located on the board, and for the upper level - a smartphone to determine the water speed. The programs take into account the peculiarities of converting data on the flow rate of water with a current meter and allow you to enter additional indicators that reduce the uncertainty of the results.
\end{abstract}

Keywords - water current meter, flow velocity measurement, Arduino programming.

\section{INTRODUCTION}

At the end of the XX and the beginning of the XXI century there are significant changes in the hydrological situation in Ukraine [1], [2] and around the world [3], [4]. Current hydrological observations of natural and artificial watercourses will allow to make up-to-date plans for the management of river basins and sub-basins, to obtain objective data on water consumption and to plan water management and environmental activities [5]. Accurate and fast measurement of instantaneous water flow velocities in open watercourses is an urgent problem. Given the critical underfunding of the water industry in Ukraine, one of the ways to obtain accurate and up-to-date information on the speed and flow of water flows in rivers and canals is to modernize existing water meters in many water management organizations, including water current meter. They are the most common devices in the history of hydrology for measuring the speed of water flows. The use of direct velocity measurement methods often provides more accurate information on the speed of water flows with varying degrees of pollution, chemical and mechanical composition, temperature and hydraulic nature of the flow.

\section{EASE OF USE}

A water current meter is a simple and reliable device in which the oncoming flow of water rotates the blades, on the shaft of which a worm gear is mounted, which reduces the number of revolutions and activates the counting mechanism. Modernization of hydrometric mills is carried out in the directions [6]-[8]:

1. Improving the shape, size, material and other properties of the blades in order to increase sensitivity, reduce inertia and flow resistance, optimize the component effect.

2. Improving the design, shape and size of the device to stabilize the direction.

3. Optimization of the principles of output signal formation.

4. Improving the counting device.

5. Improving other elements of the water current meter to improve measurement accuracy, facilitate the process and reduce measurement time, etc.

To facilitate the measurement of the water current velocity (average velocity for the stream flow and stream flow profile) in open watercourses in NUWEE, we are modernizing the water current meter GR-21M in the fourth direction. Reading the number of revolutions of the water current meter, primary data processing and determining the speed of water in open streams is performed by the Arduino Micro board. Obtaining input parameters of the water current meter, flow and the process of measuring and outputting the result is on a smartphone running Android.

The choice of the Arduino Micro board platform as the secondary measuring transducer is justified by the peculiarity of its hardware. Support directly by the microcontroller of the USB interface allows you to configure it to work in USB CDC 


\section{Modeling, control and information technologies $\mathbf{- 2 0 2 0}$}

mode, the driver of which is available in many Android smartphones, and therefore there is the ability to connect to the most common smartphones via USB OTG.

The Arduino Pro Micro board is based on the Microchip ATmega32U4 microcontroller and has a relatively small size $33 \times 18 \mathrm{~mm}$, which allows you to create convenient mobile devices based on it. The board has 24 contacts and 3 LEDs, one of which is a power indication. There are also 4 analog inputs, 5 PWM outputs, hardware UART. All this allows you to connect the Arduino Pro Micro module to the grinder through the contacts of the standard indicator light. It is not necessary to apply additional supply voltage to the contacts of the water current meter, as the pull-up to the supply voltage through the built-in pull-up resistors is used.

Measurement screw rotation of the water current meter by the Arduino Pro Micro board is performed according to the appropriate algorithms: for the lower level - the microcontroller located on the board (Fig. 1) and the upper level - the smartphone (Fig. 2). The microcontroller has the functions of detecting the fact of contact closure, measuring the time between contact closure and calculating the speed using the parameters obtained at the beginning of the program on the smartphone. The smartphone provides input of water current meter parameters, sending to the board the parameters used for calculation of speed, and output of result of measurement.

The Android application uses a library com.github.felHR85:UsbSerial:6.1.0 [9] to support the serial interface via USB. The library supports data exchange with USB CDC devices, USB-Serial converters based on Silicon Labs CP2101, CP2102, CP2103, CP2104, CP2105, CP2108, Prolific PL2303, WCH CH340, FTDI FT8U232AM, FT4232H, FT232H, FT230X, FT232RL Therefore, if you need to create multiple measuring systems, it is advisable to use a microcontroller with fewer peripherals, such as STMicroelectronics STM8S003F3, and one of the listed USBSerial converters. This reduces the total cost of the system hardware.

At the beginning of the program, the parameters of the water current meter are entered, which are registered in its accompanying documentation and determined by the results of the last calibration: geometric pitch of the water current meter screw, $k_{r}, \mathrm{~mm}$; hydraulic pitch of the screw, $k, \mathrm{~mm}$; the initial speed at which the rotor of the screw begins to rotate unevenly, $u_{0}, \mathrm{~m} / \mathrm{s}$, the minimum flow rate at which it is already possible to use the water current meter $u_{H \Lambda}, \mathrm{m} / \mathrm{s}$.

The geometric pitch of the water current meter screw depends on its geometric parameters

$$
k_{r}=\frac{2 \pi r}{\operatorname{tg} \alpha}, \mathrm{mm},
$$

where $r$ - the distance from the center of the blade of screw to its axis of rotation, $\alpha$ - the angle of inclination of the blade of screw to the area perpendicular to the axis of rotation.

The geometric pitch $k_{r}$ is slightly less than the hydraulic $k=u / n$, as it takes into account the additional resistance to rotation. That is, at the same speed $u$, the rotor in real conditions makes a smaller number of revolutions $n$ in comparison with theoretical calculations without resistance.

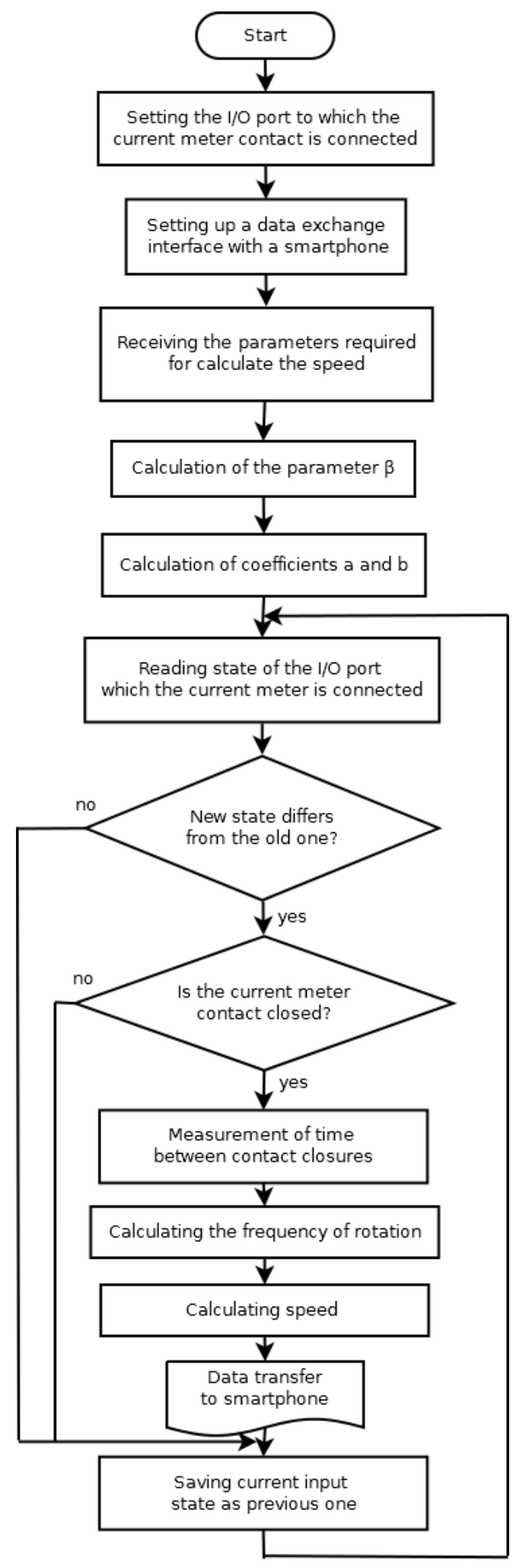

Figure 1. Block diagram of program algorithm in the board Arduino 


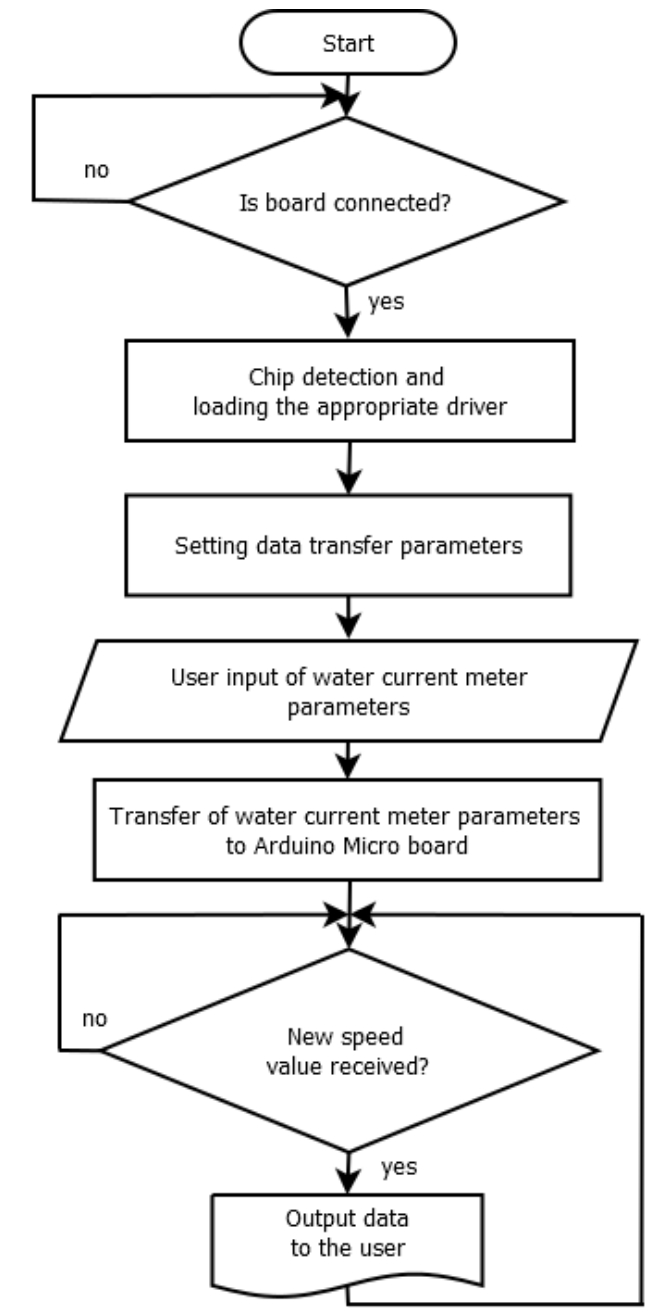

Figure 2. Block diagram of program algorithm on the smartphone

The calculation of the water flow rate depending on the number of revolutions $u(n)$ is performed according to the equation of M. Schmidt [10]

$$
u(n)=a \cdot n+\sqrt{b \cdot n^{2}+u_{0}^{2}}
$$

where $a, b$ - empirical coefficients, [10]

$$
\begin{aligned}
& a=k \cdot(0.99-\beta), \\
& b=(k \cdot \beta)^{2},
\end{aligned}
$$

where $\beta$ is an empirical parameter determined by the formula V.H. Zheleznyakov [11]

$$
\beta=6.9 \cdot u_{0}-0.06+\sqrt{\left(2.3 \cdot u_{0}-0.055\right)^{2}+0.0058}
$$

The following parameters are the limitations that reduce the uncertainty of the obtained results. At flow velocities from $u_{0}$ to $u_{H M}$, the water current meter readings are unstable due to the significant influence of mechanical resistances in the bearings, the uncertainty is more than $10 \%$, so $u_{\mu M}$ is the minimum value [11]. The limitation "from above" is the upper critical speed uvk, which for GR-21M is $8 \mathrm{~m} / \mathrm{s}$. At this velocity, due to intense vortex formation and cavitation, there is a deviation from the linear dependence $u=f(n)$.

When measuring slow flows, when $u<u_{\kappa}$ (subcritical mode) significantly affects the readings of the water current meter it is wear of the rotor bearings, getting into the moving joints of solid particles, dimensions of parts and gaps in conjugations and increasing the resistance to rotation with decreasing water temperature and, accordingly, oils in the oil chambers and contact mechanism (Fig. 3).
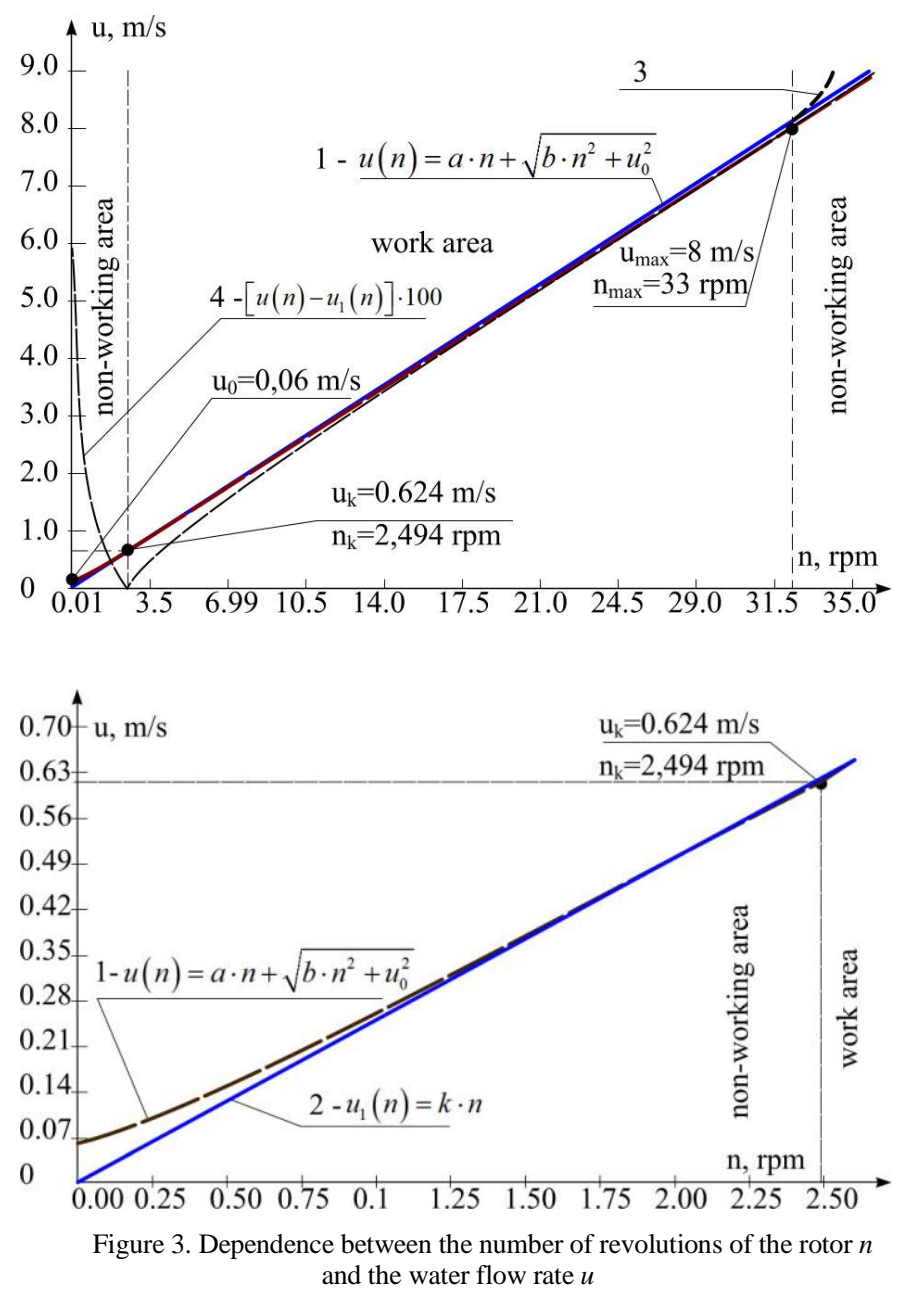

Dependence between the number of revolutions of the rotor of the screw (digital values are taken for GR-21M) of the water current meter $n$ and the water flow rate $u$ is shown in Fig. 3:

a - full range of speeds;

b - the first part of the graph, from the minimum, initial speed at which the rotor of the screw only begins to rotate unevenly, overcoming the sliding friction resistance in the bearing supports of the mill $u_{0}$ to the minimum flow rate $u_{H M}$, at which measurement uncertainty begins to meet the norms and the water current meter can be used;

$1-u(n)=a \cdot n+\sqrt{b \cdot n^{2}+u_{0}^{2}}$ - taking into account resistances; 


\section{Modeling, control and information technologies $-\mathbf{2 0 2 0}$}

2 - $u_{1}(n)=k \cdot n$ - without taking into account resistances (without taking into account the moments of sliding friction and rest friction);

3 - at high flow rates (more than $u_{\max }$ ) happens a significant increase in hydraulic resistance due to significant vortex formation at the edges of the blades and the attached masses of fluid, as well as in flows with a large amount of air, therefore, it is not recommended to measure such flows due to large inaccuracies;

4 - is the absolute value of the difference between the dependencies with and without taking into account the resistance to rotation of the rotor of the screw of the water current meter.

The critical velocity is determined by the formula V.H. Zheleznyakov [11]

$$
u_{k}=7.1 \cdot u_{0} / \sqrt{\beta}
$$

When calculating, it is also necessary to take into account the component of the water current meter - the ability to measure the component of the velocity vector in the direction of the axis of the water current meter in oblique currents. When using for GR-21M propeller №1 $0 \ldots 5 \%$, and №2 - 20.. 45\%.

After processing the results of instantaneous velocity measurements and measurements of the channel profile, this allows to determine the water flow in the watercourse.

\section{CONCLUSIONS}

Therefore, given the number of advantages of water current meter (slight dependence on temperature conditions, chemical composition of water and the presence of suspended particles in the water flow, they are quite common in water management organizations and high quality and durability of their mechanical part) they can be chosen as basic models for modernization.

It is best to use single-turn water current meter GR-99, GR101 with a magnetically controlled watertight contact (reed switch) to measure slow flows (up to $2.5 \mathrm{~m} / \mathrm{s}$ ). If it is necessary to measure fast flows (up to $8 \mathrm{~m} / \mathrm{s}$ ), GR-21M and GR-55 will be more accurate, for which $\mathrm{D} / \mathrm{k}=6$ (D - is the diameter of the blades water current meter).

They can still compete successfully with flow meters which are equipped the stationary hydrometric posts on large rivers, or portable Acoustic Doppler Profiler (ADP). The main factor that keeps them competitive is their low price, availability and broad prospects for process automation and calculation of measurement results. Having received a convenient mobile application with a detailed description of the measurement process, restrictions and purchasing an inexpensive hydrometric mill, it is possible to conduct systematic hydrometric studies of small rivers of Ukraine, drainage and irrigation canals, which is relevant in the current trend of water management.

Reduction of measurement uncertainty for flow rates below critical $u_{k}$ and close to $u_{H \mu}$ is possible with the use of modern synthetic or mineral water-resistant oils with high water resistance. Namely the following list of indicators should be provided: low solubility in water, washability, hygroscopicity, stability and stability of viscosity value at temperature changes from -10 to $+35{ }^{\circ} \mathrm{C}$. These requirements are met by a number of oils and, in particular, based on Perfluoropolyether (PFPE) [12].

In practice, during normal calibration in the nonlinear part of the $u(n)$ curve, the random component of the instrumental error of the water current meter is about $2 \%$.

In addition to the random error, the instrumental error of the water current meter includes systematic components that generate flow turbulence, which are cut off by introducing restriction $u_{\max }$.

Further improvement can be directed to the software task of the measurement protocol depending on the profile and other parameters of the bed of the channel and the visualization of the measurement process. This will further facilitate testing and create the conditions for automatic flow profile construction.

\section{REFERENCES}

[1] A. V. Jacyk, Y. M. Grishchenko, L. A. Volkova, I. A. Pashenyuk. "Water resources: use, protection, reproduction, management" . Kyiv: Genesis, 2007. (in Ukrainian).

[2] L. O. Gorbacheva, "Modern intra-annual distribution of water runoff of rivers of Ukraine” , Ukrainian Geographical Journal, vol. 3, pp. 16-23, 2015. (in Ukrainian).

[3] S. Linke et al., "Global hydro-environmental sub-basin and river reach characteristics at high spatial resolution" , Sci. Data, vol. 6, no. 1, p. 283, Dec. 2019, doi: 10.1038/s41597-019-0300-6.

[4] G. Blöschl et al., "Changing climate both increases and decreases European river floods” , Nature, vol. 573, no. 7772, pp. 108-111, Sep. 2019, doi: 10.1038/s41586-019-1495-6.

[5] S. V. Klimov, E. G. Gerasimov, and O. L. Pinchuk, “Guidelines for independent work and practical tasks in the discipline "Operational Hydrometry" for students of higher education of the second (master's) level in the specialty 194 "Hydraulic construction, water engineering and water technologies", educational program "Hydroinformatics" ",

2019.

http://ep3.nuwm.edu.ua/id/eprint/14689 (accessed Mar. 23, 2020). (in Ukrainian).

[6] D. E. Klimenko, “Development of hydrometric turntables in Russia and abroad" , Geographical Bulletin, no. 2, pp. 64-76, 2010. (in Russian).

[7] O. P. Budz, "Hydrology" . Interactive complex of educational and methodical support of the discipline. Rivne: NUVGP, 2008. 170 p. (in Ukrainian).

[8] B. V. Biryukov and M. A. Danilov, “Accurate measurements of fluid flow". Moscow: Mashinostroenie, 1977. (in Russian).

[9] GitHub - felHR85/UsbSerial: Usb serial controller for Android. https://github.com/felHR85/UsbSerial (accessed Mar. 23, 2020).

[10] M. N. Tsivin, P. I. Abramenko, "Hydrometry: Theory and Practice of Measuring the Speed of Water Flow in Open Channels" . Kiev: IGiM, 2003. 107 p. (in Russian).

[11] G. V. Zheleznyakov, "Theoretical bases of hydrometry", Hydrometeorological publishing house. Leningrad, 1968. 291 p. (in Russian).

[12] «PFPE Oils», Fluorosilicones, PFPE Oils, PTFE Micropowders, PFSA Membranes. https://www.fluorochemie.com/products/pfpelubricants (accessed Aug. 19, 2020). 